\title{
Steady and unsteady Hall magnetohydrodynamics near an X-type magnetic neutral line
}

Bhimsen K. Shivamoggi

University of Central Florida

Find similar works at: https://stars.library.ucf.edu/facultybib2010 University of Central Florida Libraries http://library.ucf.edu

This Article is brought to you for free and open access by the Faculty Bibliography at STARS. It has been accepted for inclusion in Faculty Bibliography 2010 s by an authorized administrator of STARS. For more information, please contact STARS@ucf.edu.

\section{Recommended Citation}

Shivamoggi, Bhimsen K., "Steady and unsteady Hall magnetohydrodynamics near an X-type magnetic neutral line" (2011). Faculty Bibliography 2010s. 1912.

https://stars.library.ucf.edu/facultybib2010/1912

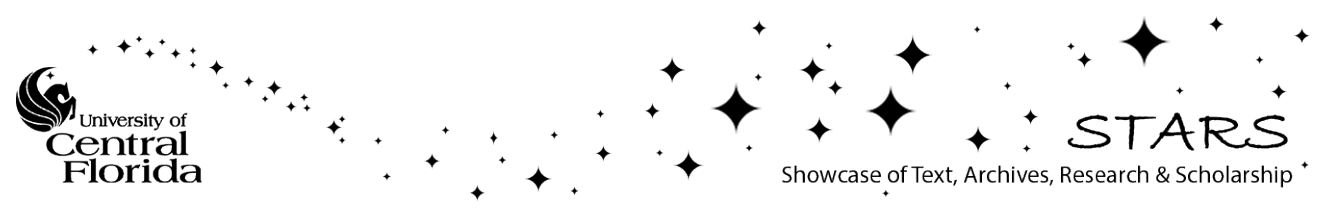


Steady and unsteady Hall magnetohydrodynamics near an X-type magnetic neutral line

Bhimsen K. Shivamoggi

Citation: Physics of Plasmas 18, 052304 (2011); doi: 10.1063/1.3581092

View online: https://doi.org/10.1063/1.3581092

View Table of Contents: http://aip.scitation.org/toc/php/18/5

Published by the American Institute of Physics

\section{Articles you may be interested in}

The role of guide field in magnetic reconnection driven by island coalescence

Physics of Plasmas 24, 022124 (2017); 10.1063/1.4976712 


\title{
Steady and unsteady Hall magnetohydrodynamics near an $X$-type magnetic neutral line
}

\author{
Bhimsen K. Shivamoggi \\ University of Central Florida, Orlando, Florida 32816-1364, USA
}

(Received 5 November 2010; accepted 9 March 2011; published online 26 May 2011)

\begin{abstract}
Hall magnetohydrodynamics (MHD) properties near a two-dimensional (2D) $X$-type magnetic neutral line in the steady state are considered via heuristic and rigorous developments. The heuristic development turns out to be useful in providing insight into the lack of dependence of the reconnection rate on the mechanism breaking the frozen-in condition of the magnetic field lines in the electron fluid. The latter result can be understood in terms of the ability of the ions and electrons to transport equal amounts of magnetic flux per unit time out of the reconnection region. The Hall effects are shown via a rigorous development to be able to sustain the hyperbolicity of the magnetic field (and hence a more open $X$-point configuration) near the neutral line in the steady state. The time-dependent Hall MHD problem shows that the Hall effect, when sufficiently strong, can indeed quench the finite-time singularity exhibited in ideal MHD. (C) 2011 American Institute of Physics. [doi:10.1063/1.3581092]
\end{abstract}

\section{INTRODUCTION}

In resistive magnetohydrodynamics (MHD), the ion inflow is the only means to transport magnetic flux into the reconnection layer. However, as the resistivity is decreased, large magnetic pressure gradients develop upstream of the reconnection layer, which start slowing the ion inflow down and reduce the magnetic flux transport into the reconnection layer (and hence the reconnection rate)- the so-called pressure problem $\left(\mathrm{Clark}^{1}\right)$. The Hall effect $\left(\right.$ Sonnerup $\left.{ }^{2}\right)$ can overcome the pressure problem (Dorelli and Birn ${ }^{3}$ and Knoll and Chacón $^{4}$ ), thanks to the decoupling of electrons from ions on length scales below the ion skin depth $d_{i}$. So, if the reconnection layer width is less than $d_{i}$, the electron inflow can keep on going which continually transports the magnetic flux into the reconnection layer and hence reduces the flux pile-up. Previous numerical work (Shay et al., ${ }^{5}$ Rogers et al., ${ }^{6}$ Fitzpatrick, ${ }^{7}$ and Knoll and Chacón) ${ }^{4}$ indicated that the dissipation region in Hall MHD, as $d_{i}$ increases, changes from an elongated current-sheet geometry (Sweet ${ }^{8}$ and Parker $^{9}$ type) to a more open $X$-point geometry (Petschek ${ }^{10}$ type). However, recent fully kinetic simulations (Daughton et al. ${ }^{11}$ and Karimabadi et al. $^{12}$ ) and electron MHD (EMHD)-based treatments (Chacón et al. ${ }^{13}$ ) have shown that the elongated current sheets are also possible. On the other hand, more recent particle-in-cell (PIC) simulations (Shay et al. ${ }^{14}$ ) show spatial localization of the out-of-plane current to within a few $d_{i} s$ of the $X$-line. Recent laboratory experiments (Frank et $a l .{ }^{15}$ ) also reported the appearance of an $X$-type magnetic neutral line in Hall MHD. In an effort to shed further light on this issue, we consider in this paper Hall MHD properties near a two-dimensional (2D) $X$-type magnetic neutral line in the steady state via heuristic and rigorous developments and investigate whether or not the Hall effects favor the hyperbolicity of the magnetic field near the neutral line. In the special Hall nonresistive or inviscid case, certain ambiguities (which are peculiar to the Hall MHD case) present themselves, which may be resolved upon connecting the steady Hall MHD state with an equilibrium solution of the corresponding time-dependent Hall MHD problem.

In fact, when a plasma collapses near the neutral line of the applied magnetic field the continual accumulation of the magnetic flux in the region of the neutral sheet puts the current sheet in a nonstationary state (Syrovatskii ${ }^{16}$ ). Extensive theoretical studies have been done on the various aspects of time-dependent 2D MHD flow of an incompressible plasma near an $X$-type magnetic neutral line (Uberoi, ${ }^{17}$ Chapman and Kendall, ${ }^{18}$ Shivamoggi, ${ }^{19,20}$ and Rollins and Shivamoggi ${ }^{21,22}$ ). These formulations have described a sequence of events associated with the evolution of a current sheet near an $X$-type magnetic neutral line in agreement with laboratory experiments $\left(\right.$ Frank $\left.^{23}\right)$. They have also provided an account of the significant effect that plasma density variations near the magnetic neutral point have on the currentsheet formation process there in agreement with numerical results (Brunnel et al. $^{24}$ ). An investigation of unsteady Hall MHD near an $X$-type magnetic neutral line (Shivamoggi ${ }^{25}$ ) is then in order, its potential connection with the corresponding steady Hall MHD problem notwithstanding. (Unsteady Hall MHD near an $X$-type magnetic neutral line was also recently considered by Litvinenko; ${ }^{26}$ his solution is rather different from the present unsteady solution.)

\section{GOVERNING EQUATIONS FOR HALL MHD}

Consider an incompressible, two-fluid, quasineutral plasma. The equations governing this plasma dynamics are (in usual notation)

$$
\begin{aligned}
n m_{e}\left[\frac{\partial \mathbf{v}_{e}}{\partial t}+\left(\mathbf{v}_{e} \cdot \nabla\right) \mathbf{v}_{e}\right]= & -\nabla p_{e}-n e\left(\mathbf{E}+\frac{1}{c} \mathbf{v}_{e} \times \mathbf{B}\right) \\
& +n e \eta \mathbf{J}
\end{aligned}
$$




$$
\begin{aligned}
n m_{i}\left[\frac{\partial \mathbf{v}_{i}}{\partial t}+\left(\mathbf{v}_{i} \cdot \nabla\right) \mathbf{v}_{i}\right] & =-\nabla p_{i}+n e\left(\mathbf{E}+\frac{1}{c} \mathbf{v}_{i} \times \mathbf{B}\right) \\
& -n e \eta \mathbf{J}, \\
\nabla \cdot \mathbf{v}_{e} & =0 \\
\nabla \cdot \mathbf{v}_{i} & =0 \\
\nabla \cdot \mathbf{B} & =0 \\
\nabla \times \mathbf{B} & =\frac{1}{c} \mathbf{J} \\
\nabla \times \mathbf{E} & =-\frac{1}{c} \frac{\partial \mathbf{B}}{\partial t}
\end{aligned}
$$

where

$$
\mathbf{J} \equiv n e\left(\mathbf{v}_{i}-\mathbf{v}_{e}\right) .
$$

Neglecting electron inertia ( $\left.m_{e} \Rightarrow 0\right)$, Eqs. (1) and (2) can be combined to give an ion-fluid equation of motion,

$$
n m_{i}\left[\frac{\partial \mathbf{v}_{i}}{\partial t}+\left(\mathbf{v}_{i} \cdot \nabla\right) \mathbf{v}_{i}\right]=-\nabla\left(p_{i}+p_{e}\right)+\frac{1}{c} \mathbf{J} \times \mathbf{B},
$$

and a generalized Ohm's law

$$
\mathbf{E}+\frac{1}{c} \mathbf{v}_{i} \times \mathbf{B}=\eta \mathbf{J}+\frac{1}{n e c} \mathbf{J} \times \mathbf{B} .
$$

Nondimensionalize distance with respect to a typical length scale $a$, magnetic field with respect to a typical magnetic field strength $B_{0}$, time with respect to the reference Alfvén time $\tau_{A} \equiv a / V_{A_{0}}$, where $V_{A_{0}} \equiv B_{0} / \sqrt{m_{i} n}$, and introduce the magnetic and ion-fluid velocity stream functions according to

$$
\left.\begin{array}{rl}
\mathbf{B} & =\nabla \psi \times \hat{\mathbf{i}}_{z}+b \hat{\mathbf{i}}_{z} \\
\mathbf{v}_{i} & =\nabla \phi \times \hat{\mathbf{i}}_{z}+w \hat{\mathbf{i}}_{z}
\end{array}\right\},
$$

and assume the physical quantities of interest have no variation along the $z$-direction. Here, $\hat{\mathbf{i}}_{z}$ is the unit vector in the $z$-direction. The Hall magnetic field $b$ is believed to be produced by the dragging of the in-plane magnetic field in the out-of-plane direction by the electrons near the $X$-type magnetic neutral line (Shay et al. ${ }^{5,14}$ ). Equations (1) and (2) then yield

$$
\begin{gathered}
\frac{\partial \psi}{\partial t}+[\psi, \phi]+\sigma[b, \psi]=\hat{\eta} \nabla^{2} \psi, \\
\frac{\partial b}{\partial t}+[b, \phi]+\sigma\left[\psi, \nabla^{2} \psi\right]+[\psi, w]=\hat{\eta} \nabla^{2} b, \\
\frac{\partial}{\partial t}\left(\nabla^{2} \phi\right)+\left[\nabla^{2} \phi, \phi\right]=\left[\nabla^{2} \psi, \psi\right], \\
\frac{\partial w}{\partial t}+[w, \phi]=[b, \psi],
\end{gathered}
$$

where

$$
[A, B] \equiv \nabla A \times \nabla B \cdot \hat{\mathbf{i}}_{z}, \sigma \equiv \frac{d_{i}}{a}, d_{i} \equiv \frac{c}{\omega_{p i}}, \hat{\eta} \equiv \frac{\eta c^{2} \tau_{A}}{a^{2}} .
$$

For the system of Eqs. (12)-(15) in the ideal limit, the conservation of magnetic flux is replaced by the conservation of generalized ion magnetic flux, which is the magnetic flux augmented by the ion-fluid vorticity flux (Shivamoggi ${ }^{27}$ ).

\section{A HEURISTIC ANALYSIS}

It is instructive to do a heuristic analysis (Shivamoggi ${ }^{28}$ ) to develop an estimate on the geometry of the dissipation region prior to a more rigorous formulation. Let the dissipation region has a length $L$ in the outflow $x$-direction and a width $\delta$ in the inflow $y$-direction.

We then have from Eq. (5),

$$
\frac{B_{x}}{L} \sim \frac{B_{y}}{\delta} .
$$

We have from the $z$-component of Eq. (10),

$$
\eta J_{z} \sim \frac{1}{n e c} J_{x} B_{y},
$$

and on using Eqs. (6), (17) becomes

$$
\eta c \frac{B_{x}}{\delta} \sim \frac{1}{n e c} c \frac{B_{z}}{\delta} B_{y}
$$

or

$$
\eta B_{x} \sim \frac{1}{n e c} B_{y} B_{z} .
$$

Next, the $z$-component of the curl of Eq. (10) gives

$$
\eta c \nabla^{2} B_{z}=\frac{1}{n e c}(\mathbf{B} \cdot \nabla) J_{z},
$$

from which

$$
\eta c \frac{B_{z}}{\delta^{2}} \sim \frac{1}{n e c} \frac{B_{x}}{L} c \frac{B_{x}}{\delta}
$$

and on using (16), (20) becomes

$$
\eta \frac{B_{z}}{\delta^{2}} \sim \frac{1}{n e c} \frac{B_{y}}{\delta} \frac{B_{x}}{\delta}
$$

or

$$
\eta B_{z} \sim \frac{1}{n e c} B_{x} B_{y} .
$$

(18) and (21) give

$$
B_{z} \sim B_{x} .
$$

Using (22), (18) gives

$$
B_{y} \sim n e c \eta .
$$

Using (16), (23) leads to

$$
\frac{\delta}{L} B_{x} \sim \text { nec } \eta .
$$


On rearranging, (24) gives

$$
\frac{\delta}{L} \sim \frac{1}{\sigma S}
$$

where

$$
S \equiv \frac{V_{A_{i}} a}{\tilde{\eta}}, \tilde{\eta} \equiv \eta c^{2}, V_{A_{i}} \equiv \frac{B_{x}}{\sqrt{n m_{i}}} .
$$

Equation (25) was also given by Chacón et al. ${ }^{13}$ by using a more rigorous formulation.

Noting that in the Hall resistive regime

$$
\sigma>1, S \gg 1
$$

Eq. (25) implies that the diffusion region in the Hall resistive region may be expected to be elongated. Rigorous formulation in Sec. IV, on the other hand, shows that this result represents only part of the story because the heuristic development fails to recognize the steady state as the asymptotic limit of the corresponding time-dependent problem. The latter aspect makes the actual story more complicated than what is conveyed by the heuristic development.

It is interesting to note, however, that the above heuristic analysis sheds some light on the conjecture (Mandt et al. ${ }^{29}$ and Shay and Drake ${ }^{30}$ ) that the reconnection rate in Hall MHD is primarily controlled by ions (which are decoupled from the electrons) and is independent of the mechanism that breaks the frozen-in condition of the magnetic field lines in the electron fluid (resistivity or electron inertia).

For the Hall-resistive case, the reconnection rate is given by

$$
E \sim \eta J_{z}
$$

and on using Eqs. (6) and (23), (26) becomes

$$
E \sim \frac{B_{y}}{n e c} c \frac{B_{x}}{\delta}
$$

Using (16), (27) becomes (Shivamoggi, ${ }^{28}$ Simakov and Chacón, ${ }^{31}$ and Malyshkin ${ }^{32}$ )

$$
E \sim \frac{B_{x}^{2}}{n e L}
$$

It may be noted that (28) is in agreement with the estimate for the electron outflow velocity given by Mandt et al. ${ }^{29}$ for the Hall-resistive case,

$$
V_{e_{x}} \sim \frac{\Omega_{i} d_{i}^{3}}{L^{2}}
$$

On the other hand, if we consider the electron inertia to constitute the mechanism that breaks the frozen-in condition of the magnetic field lines, the Ohm's law now takes the form (Coppi et al. $^{33}$ )

$$
\mathbf{E}+\frac{1}{c} \mathbf{v}_{e} \times \mathbf{B}=\frac{1}{\omega_{p_{e}}^{2}} \frac{d \mathbf{J}}{d t} .
$$

In the electron-inertia case, the reconnection rate is therefore given by

$$
E \sim \frac{1}{\omega_{p_{e}^{2}}} \frac{d J_{z}}{d t}
$$

Using Eq. (6), (31) may be rewritten as

$$
E \sim \frac{1}{\omega_{p_{e}^{2}}} \frac{1}{\tau_{A_{e}}} \frac{B_{x}}{\delta}
$$

where

$$
\tau_{A_{e}} \sim \frac{L}{V_{A_{e}}}, V_{A_{e}} \equiv \frac{B_{x}}{\sqrt{n m_{e}}} .
$$

Taking $\delta \sim d_{e}$, (32) becomes (Shivamoggi, ${ }^{28}$ Zocco et al., ${ }^{34}$ and Malyshkin ${ }^{35}$ )

$$
E \sim \frac{B_{x}^{2}}{n e L}
$$

which is the same as the one, namely, (28), for the Hall resistive case! This appears to support the conjecture (Mandt et al. $^{29}$ and Shay and Drake ${ }^{30}$ ) that the reconnection rate is independent of the mechanism that breaks the frozen-in condition of the magnetic field lines in the electron fluid. A similar conclusion was reached by Chacón et al. ${ }^{13}$ (see also Sullivan et $a l .{ }^{36}$ ) who considered the electron hyper-resistivity to constitute another mechanism that breaks the frozen-in condition of the magnetic field lines in the electron fluid.

It is of interest to note that (28) and (33) may be rewritten as

$$
E \sim \frac{B_{x}^{2}}{n e L} \sim B_{x}\left(\frac{V_{A_{i}}}{c}\right)\left(\frac{d_{i}}{L}\right) \sim B_{x}\left(\frac{V_{A_{e}}}{c}\right)\left(\frac{d_{e}}{L}\right) .
$$

Equation (34) shows that the lack of dependence of the reconnection rate on the mechanism breaking the frozen-in condition of the magnetic field lines can be understood in terms of the ability of the ions and electrons to transport equal amounts of magnetic flux per unit time out of the reconnection region. The insensitivity of the reconnection rate, according to (34), on the particle mass has been confirmed by the recent particlein-cell simulations (Shay et $a l .{ }^{14}$ ). We are assuming here, as confirmed by the recent numerical simulations (Drake et al. ${ }^{37}$ ) that, in the electron-inertia case, the outflow velocity of the electrons from the dissipation region is given by the Alfvén speed based on the upstream magnetic field $B_{x}$ with the width of the dissipation region scaling with $d_{e}$.

\section{STEADY-STATE PROPERTIES NEAR AN $X$-TYPE NEUTRAL LINE}

Consider Hall MHD properties near a 2D X-type magnetic neutral line in the steady state. Equations (12)-(15) now become

$$
\begin{aligned}
& -c E+\psi_{10} \phi_{01}-\psi_{01} \phi_{10}+\sigma\left(b_{10} \psi_{01}-b_{01} \psi_{10}\right) \\
& =\hat{\eta}\left(\psi_{20}+\psi_{02}\right)
\end{aligned}
$$




$$
\begin{aligned}
& b_{10} \phi_{01}-b_{01} \phi_{10}+\sigma\left[\psi_{10}\left(\psi_{21}+\psi_{03}\right)-\psi_{01}\left(\psi_{30}+\psi_{12}\right)\right] \\
& \quad+\psi_{10} w_{01}-\psi_{01} w_{10}=\hat{\eta}\left(b_{20}+b_{02}\right), \\
& \phi_{10}\left(\phi_{21}+\phi_{03}\right)-\phi_{01}\left(\phi_{30}+\phi_{12}\right)-\left[\psi_{10}\left(\psi_{21}+\psi_{03}\right)\right. \\
& \left.\quad+\psi_{01}\left(\psi_{30}+\psi_{12}\right)\right]=-v\left(\phi_{40}+2 \phi_{22}+\phi_{04}\right), \\
& w_{10} \phi_{01}-w_{01} \phi_{10}+\psi_{10} b_{01}-\psi_{01} b_{10}=v\left(w_{20}+w_{02}\right),
\end{aligned}
$$

where

$$
F_{m n} \equiv \frac{\partial^{m+n} F}{\partial x^{m} \partial y^{n}}, \quad E \equiv-\frac{1}{c} \frac{\partial \psi}{\partial t}
$$

and we have now included in Eqs. (14) and (15) viscous effects in the plasma, which become important near the magnetic neutral line (Tsuda and $\mathrm{Ugai}^{38}$ ); $v$ is the ion viscosity coefficient.

Following Cowley ${ }^{39}$ and Shivamoggi, ${ }^{40}$ let us expand the velocity and magnetic fields in a Taylor series about the neutral line taken to be at $x=0, y=0$. Equations (35)-(38) may then be used to derive relationships between the coefficients of the series. The latter are simply the partial derivatives of the velocity and magnetic fields at the neutral line. Motivated by the symmetry properties of Eqs. (35)-(38) in the ideal limit, we may consider $\psi$ and $w$ to be even functions of both $x$ and $y$, and $\phi$ and $b$ to be odd functions of both $x$ and $y$ (this also enables the out-of-plane magnetic field $b$ to exhibit the quadrupolar structure (Terasawa ${ }^{41}$ ) characteristic of Hall MHD_this has also been confirmed by laboratory experiments (Ren et al. ${ }^{42}$ ), in situ measurements in the magnetotail (Fujimoto et al., ${ }^{43}$ Nagai et al., ${ }^{44}$ and Oieroset et $a l .{ }^{45}$ ) and theoretical developments (Shivamoggi ${ }^{46}$ ). Thus, we write

$$
\begin{gathered}
\psi=\sum_{m} \sum_{n} \Psi_{2 m, 2 n} \frac{x^{2 m} y^{2 n}}{(2 m) !(2 n) !} \\
\phi=\sum_{m} \sum_{n} \Phi_{2 m+1,2 n+1} \frac{x^{2 m+1} y^{2 n+1}}{(2 m+1) !(2 n+1) !} \\
b=\sum_{m} \sum_{n} B_{2 m+1,2 n+1} \frac{x^{2 m+1} y^{2 n+1}}{(2 m+1) !(2 n+1) !} \\
w=\sum_{m} \sum_{n} W_{2 m, 2 n} \frac{x^{2 m} y^{2 n}}{(2 m) !(2 n) !} .
\end{gathered}
$$

Equation (39) reflects the fact that the origin in the $x, y$-plane is both the $X$-type neutral point and a stagnation point of the flow.

Using (39), Eqs. (35) and (38) give, on evaluation at the origin,

$$
\begin{gathered}
\hat{\eta}\left(\Psi_{20}+\Psi_{02}\right)=-c E, \\
v\left(W_{20}+W_{02}\right)=0 .
\end{gathered}
$$

Equation (40) shows that at least one of $\Psi_{20}$ and $\Psi_{02}$ must be nonzero. It turns out indeed, as we will see on the following, that neither of them can be zero.

Let us differentiate Eqs. (35) and (38) with respect to $x$ and $y$ separately and differentiate the resulting four equa- tions, respectively, with respect to $x, y$ and $x, y$. We then obtain

$$
\begin{aligned}
& \psi_{30} \phi_{01}+2 \psi_{20} \phi_{11}+\psi_{10} \phi_{21}-\psi_{21} \phi_{10}-2 \psi_{11} \phi_{20}-\psi_{01} \phi_{30} \\
& +\sigma\left(b_{30} \psi_{01}+2 b_{20} \psi_{11}+b_{10} \psi_{21}-b_{21} \psi_{10}-2 b_{11} \psi_{20}-b_{01} \psi_{30}\right) \\
& =\hat{\eta}\left(\psi_{40}+\psi_{22}\right), \\
& \psi_{12} \phi_{01}+2 \psi_{11} \phi_{02}+\psi_{10} \phi_{03}-\psi_{03} \phi_{10}-2 \psi_{02} \phi_{11}-\psi_{01} \phi_{12} \\
& +\sigma\left(b_{12} \psi_{01}+2 b_{11} \psi_{02}+b_{10} \psi_{03}-b_{03} \psi_{10}-2 b_{02} \psi_{11}-b_{01} \psi_{12}\right) \\
& =\hat{\eta}\left(\psi_{22}+\psi_{04}\right),
\end{aligned}
$$

$w_{30} \phi_{01}+2 w_{20} \phi_{11}+w_{10} \phi_{21}-w_{21} \phi_{10}-2 w_{11} \phi_{20}-w_{01} \phi_{30}$

$+\psi_{30} b_{01}+2 \psi_{02} b_{11}+\psi_{10} b_{21}-\psi_{21} b_{10}-2 \psi_{11} b_{20}-\psi_{01} b_{30}$

$=v\left(w_{40}+w_{22}\right)$,

$w_{12} \phi_{01}+2 w_{11} \phi_{02}+w_{10} \phi_{03}-w_{03} \phi_{10}-2 w_{02} \phi_{11}-w_{01} \phi_{12}$

$$
+\psi_{12} b_{01}+2 \psi_{11} b_{02}+\psi_{10} b_{03}-\psi_{03} b_{10}-2 \psi_{02} b_{11}
$$$$
-\psi_{01} b_{12}=v\left(w_{22}+w_{04}\right) \text {. }
$$

Next, let us differentiate both Eqs. (36) and (37) with respect to $x$ and then both with respect to $y$. We then obtain

$$
\begin{aligned}
b_{21} & \phi_{01}+b_{20} \phi_{02}+b_{10} \phi_{12}-b_{12} \phi_{10}-b_{02} \phi_{20}-b_{01} \phi_{21} \\
& +\sigma\left[\psi_{21}\left(\psi_{21}+\psi_{03}\right)+\psi_{20}\left(\psi_{22}+\psi_{04}\right)+\psi_{10}\left(\psi_{32}+\psi_{14}\right)\right. \\
& \left.-\psi_{12}\left(\psi_{30}+\psi_{12}\right)-\psi_{02}\left(\psi_{40}+\psi_{22}\right)-\psi_{01}\left(\psi_{41}+\psi_{23}\right)\right] \\
& +\psi_{21} w_{01}+\psi_{20} w_{02}+\psi_{10} w_{12}-\psi_{12} w_{10}-\psi_{02} w_{20} \\
& -\psi_{01} w_{21}=\hat{\eta}\left(b_{31}+b_{13}\right), \\
\phi_{21} & \left(\phi_{21}+\phi_{03}\right)+\phi_{20}\left(\phi_{22}+\phi_{04}\right)+\phi_{10}\left(\phi_{32}+\phi_{14}\right) \\
& -\phi_{12}\left(\phi_{30}+\phi_{12}\right)-\phi_{02}\left(\phi_{40}+\phi_{22}\right)-\phi_{01}\left(\phi_{41}+\phi_{23}\right) \\
& =\psi_{21}\left(\psi_{21}+\psi_{03}\right)+\psi_{20}\left(\psi_{22}+\psi_{04}\right)+\psi_{10}\left(\psi_{32}+\psi_{14}\right) \\
& -\psi_{12}\left(\psi_{30}+\psi_{12}\right)-\psi_{02}\left(\psi_{40}+\psi_{22}\right)-\psi_{01}\left(\psi_{41}+\psi_{23}\right) \\
& -v\left(\phi_{51}+2 \phi_{33}+\phi_{15}\right) .
\end{aligned}
$$

We now use (39) and evaluate Eqs. (42)-(47) at the origin,

$$
\begin{gathered}
\hat{\eta}\left(\Psi_{40}+\Psi_{22}\right)=2 \Psi_{20}\left(\Phi_{11}-\sigma B_{11}\right), \\
\hat{\eta}\left(\Psi_{22}+\Psi_{04}\right)=-2 \Psi_{02}\left(\Phi_{11}-\sigma B_{11}\right), \\
\hat{\eta}\left(B_{31}+B_{13}\right)=\sigma\left[\Psi_{20}\left(\Psi_{22}+\Psi_{04}\right)-\Psi_{02}\left(\Psi_{40}+\Psi_{22}\right)\right] \\
+\Psi_{20} W_{02}-\Psi_{02} W_{20}, \\
\Psi_{20}\left(\Psi_{22}+\Psi_{04}\right)-\Psi_{02}\left(\Psi_{40}+\Psi_{22}\right)=v\left(\Phi_{51}+2 \Phi_{33}+\Phi_{15}\right), \\
v\left(W_{40}+W_{22}\right)=2 W_{20} \Phi_{11}+2 \Psi_{02} B_{11}, \\
v\left(W_{22}+W_{04}\right)=-2 W_{02} \Phi_{11}-2 \Psi_{20} B_{11} .
\end{gathered}
$$

Using Eqs. (48) and (49), Eq. (51) gives

$$
4\left(\Phi_{11}-\sigma B_{11}\right) \Psi_{20} \Psi_{02}=-v \hat{\eta}\left(\Phi_{51}+2 \Phi_{33}+\Phi_{15}\right) .
$$

Equation (54) shows that, in the MHD resistive viscous case $(\sigma=0, \hat{\eta}$ and $v \neq 0)$, one has

$$
\Psi_{20} \neq 0, \Psi_{02} \neq 0,
$$


so the magnetic field configuration to the lowest order can be an $X$-type (Shivamoggi). ${ }^{40}$ This result continues to hold when Hall effects are included $\left(B_{11} \neq 0\right.$ - Hall effects materialize only via their signature-the quadrupolar out-ofplane magnetic field pattern). On the other hand, in the special Hall inviscid or nonresistive case, further deductions from Eq. (54) become difficult because of the factor containing the Hall contribution on the left hand side. In order to resolve the ambiguity introduced by this factor for this special Hall case, one approach is to go outside the framework of the steady Hall MHD problem and connect the steady state with an equilibrium solution of the corresponding timedependent Hall MHD problem.

\section{UNSTEADY-STATE PROPERTIES NEAR AN $X$-TYPE NEUTRAL LINE}

Let us now consider the time-dependent Hall MHD near a 2D $X$-type magnetic neutral line. We write the ion-fluid velocity as

$$
\mathbf{v}_{i}=\left(\hat{\mathbf{i}}_{z} \times \mathbf{v}_{i}\right) \times \hat{\mathbf{i}}_{z}+w \hat{\mathbf{i}}_{z} \equiv \mathbf{v}+w \hat{\mathbf{i}}_{z}
$$

Equations (9) and (10) now yield

$$
\begin{gathered}
{\left[\frac{\partial}{\partial t}+(\mathbf{v} \cdot \nabla)\right] \mathbf{v}=-\nabla P-\left(\nabla^{2} \psi\right) \nabla \psi,} \\
{\left[\frac{\partial}{\partial t}+(\mathbf{v} \cdot \nabla)\right] \psi+\sigma[b, \psi]=\hat{\eta} \nabla^{2} \psi,} \\
{\left[\frac{\partial}{\partial t}+(\mathbf{v} \cdot \nabla)\right] b+\sigma\left[\psi, \nabla^{2} \psi\right]+[\psi, w]=\hat{\eta} \nabla^{2} b,} \\
{\left[\frac{\partial}{\partial t}+(\mathbf{v} \cdot \nabla)\right] w-[b, \psi]=0 .}
\end{gathered}
$$

where

$$
P \equiv p_{e}+p_{i}+b^{2}
$$

Consider the initial-value problem for Eqs. (57)-(60) near an $X$-type magnetic neutral line with initial conditions,

$$
\begin{aligned}
t=0: v_{x}=-\dot{\gamma}_{0} x, v_{y} & =\dot{\gamma}_{0} y, \quad w=\frac{-\left(k x^{2}-y^{2}\right)}{\sigma}, \\
\psi=k x^{2}-y^{2}, \quad b & =\dot{C}_{0} x y
\end{aligned}
$$

where $\dot{\gamma}_{0}, k$, and $\dot{C}_{0}$ are externally determined parameters with $\dot{\gamma}_{0}>0, k>0$, and $\dot{C}_{0}>0$. The current density $\mathbf{J}$ corresponding to this initial condition is given by

$$
\mathbf{J}_{0}=\dot{C}_{0} x \hat{\mathbf{i}}_{x}-\dot{C}_{0} y \hat{\mathbf{i}}_{y}+2(1-k) \hat{\mathbf{i}}_{z}
$$

So we require $k \neq 1$ in order to make the out-of-plane component of J not vanish. This initial condition describes a stagnation-point plasma flow impinging transversely onto the $x=0$ plane and incorporates the solenoidal field constraints on $\mathrm{v}$ and $\mathrm{B}$. The spatial structure for the out-of-plane magnetic field described by this initial condition is again in rec- ognition of the quadrupolar out-of-plane magnetic field $b$ pattern characterizing the Hall effects.

Let us assume that the solution, for $t>0$, of equations and (57)-(60) with the above initial conditions is of the selfsimilar form

$$
\begin{aligned}
& v_{x}(x, y, t)=-\dot{\gamma}(t) x, v_{y}(x, y, t)=\dot{\gamma}(t) y \\
& w(x, y, t)=\frac{1}{\sigma}\left[\beta(t) y^{2}-k \alpha(t) x^{2}\right] \\
& \psi(x, y, t)=k \alpha(t) x^{2}-\beta(t) y^{2}, k>0, \\
& b(x, y, t)=\dot{C}(t) x y \\
& P(x, y, t)=-\frac{1}{2} v(t)\left(x^{2}+y^{2}\right)+P_{0}, v(t)>0
\end{aligned}
$$

with

$$
t=0: \alpha=\beta=1, \dot{\gamma}=\dot{\gamma}_{0}, \dot{C}=\dot{C}_{0}
$$

For the solution (63), $\nabla^{2} \psi=f(t)$ and $\nabla^{2} b=0$, so the effect of resistivity in this case is to add a function of $t$ to $\psi$ (which leaves the magnetic field unaltered) and hence to introduce an electric field along the $\mathrm{z}$-axis. We therefore drop the resistivity in the following. Further, for an incompressible plasma, the pressure does not have a dynamical role and is forced to be an enslaved variable in the sense that its form is chosen so as to be compatible with Eqs. (57)-(60), given the Ansätze for $v_{x}, v_{y}, w, \psi$, and $b$. In the generic situation, it may be mentioned that there are six parameters - two for the magnetic flux function $\psi$, one for the out-of-plane magnetic field $b$, and three for the velocity field $(\mathbf{v}, w)$, to be determined by only five scalar equations (57)-(60), so there is nonuniqueness in the solution. This is resolved by specifying the parameters as in (63) to close the system, so (63) is one exact solution. However, this exact solution turns out to have considerable physical significance, as discussed in the following. (It may be noted that Litvinenko ${ }^{26}$ sets up the solution and the initial conditions for $w$ and $b$ rather different from those prescribed in (63) and (64); in Litvinenko's solution the amplitude of the driving plasma flow velocity field is taken to be time independent, i.e., $\dot{\gamma}=$ const.)

Substituting (63) into Eq. (57), we obtain

$$
\ddot{\gamma}=2\left(k^{2} \alpha^{2}-\beta^{2}\right) \text {. }
$$

Equation (58) gives

$$
\begin{aligned}
& \dot{\alpha}-2(\dot{\gamma}+\sigma \dot{C}) \alpha=0, \\
& \dot{\beta}+2(\dot{\gamma}+\sigma \dot{C}) \beta=0,
\end{aligned}
$$

while Eq. (59) yields

$$
\ddot{C}=0 \text { or } \dot{C}(t)=\text { const }=\dot{C}_{0} .
$$

Equation (60) is identically satisfied by the solution Ansätze (63).

We have from Eqs. (65)-(67),

$$
\begin{gathered}
\alpha(t)=e^{2(\gamma+\sigma C)}, \\
\beta(t)=e^{-2(\gamma+\sigma C)},
\end{gathered}
$$




$$
\dot{\gamma}^{2}+2 \sigma \dot{C} \dot{\gamma}=\left[k^{2} e^{4(\gamma+\sigma C)}+e^{-4(\gamma+\sigma C)}\right]-A,
$$

with

$$
t=0: \gamma=0 \text {. }
$$

Equation (71) along with the initial conditions (64) and (72) yields

$$
A=\left(k^{2}+1\right)-\dot{\gamma}_{0}^{2}-2 \sigma \dot{C}_{0} \dot{\gamma}_{0} .
$$

It may be noted that (69) and (70) are consistent with the ion-fluid incompressibility condition

$$
\frac{d}{d t}\left(\frac{1}{2} \ln [\alpha(t) \beta(t)]\right)=\nabla \cdot \mathbf{v}=0,
$$

which is derivable from Eq. (57) on substituting (63).

Equations (65)-(67) show that the finite-time singularity exhibited in ideal MHD (Shivamoggi ${ }^{47}$ ) is quenched by the Hall effect, when sufficiently strong. This result may be appreciated by noting that Eqs. (69)-(71) lead to the exact invariant

$$
(\dot{\gamma}+\sigma \dot{C})^{2}-\left(k^{2} \alpha^{2}+\beta^{2}\right)=\text { const. }
$$

Equation (75) clearly shows the suppression of the plasma collapse process near an $X$-type magnetic neutral line in Hall MHD, when Hall effects are sufficiently strong $(\sigma \dot{C} \gg|\dot{\gamma}|)$. Physically, the suppression of the plasma collapse process near an $X$-type magnetic neutral point in Hall MHD appears to be traceable in a broad sense to additional transport mechanisms for the magnetic field due to Hall currents that accompany slippage of the magnetic field lines relative to the ion fluid. [Such a mechanism in fluids which arises due to the baroclinic effect of fluid compressibility is known to sustain the vortex reconnection process (Shivamoggi ${ }^{48}$ ).] This aspect is also reflected in the enhancement of the tearing-mode growth rate by the Hall effects (Terasawa ${ }^{41}$ and Shivamoggi ${ }^{49}$ ). More specifically, this may be traced to dispersive whistler standing-wave activity near the magnetic neutral point as per the numerical simulations (Shay et $a l .^{5,14}$ ) which linked a more open $X$-point magnetic field configuration to this activity. [Whistler waves are the characteristic modes of the system of Eqs. (12)-(15) and are implicit in the underlying dynamics (Shay et al. ${ }^{50}$ ); whistler waves near the $X$-point become standing waves because the wave velocity drops to zero there.] Dispersive whistler wave activity has also been known to lead to current-sheet broadening as per laboratory experiments (Urrutia et al..$^{51}$ ) and satellite observations at the magnetopause and the magnetotail sheet (Sonnerup et al..$^{52}$ and Fairfield et al. ${ }^{53}$ ).

Equations (66) and (67) show that the unsteady Hall system admits an equilibrium solution

$$
\dot{\gamma}=-\sigma \dot{C} \text {. }
$$

The stability of this solution can be insured upon including a higher-degree term (which may be viewed as a next term in the Taylor expansion near the magnetic neutral point) for the magnetic stream function $\psi$ and prescribing the parameter $k$ appropriately (see Appendix A).

It may be noted that the equilibrium solution (76), in conjunction with Eq. (65), would correspond to $J_{z}=0$. Inclusion of a higher-degree term again in $\psi$, would, as indicated by (A3) in Appendix A, make $J_{z} \neq 0$ and hence rectify this anomaly. The runaway behavior of the time-dependent solutions, as indicated by (75), also appears to be rather weakened by including a higher-degree term in $\psi$ (Appendix A).

Now, connecting the equilibrium solution (76) of the time-dependent Hall MHD problem with the steady HallMHD development given by (39), we have

$$
\Phi_{11}-\sigma B_{11}=0
$$

The steady Hall MHD result (54) then shows that, even in the inviscid limit $(v \Rightarrow 0)$, Eq. (55), thanks to Hall effects, continues to be valid. So, this Hall MHD result appears to be robust with respect to ion-viscosity. The hyperbolicity of the magnetic field (and hence a more open $X$-point configuration) near the neutral line is therefore sustained in the steady Hall MHD state. This appears to be traceable, as per Shay et al., ${ }^{5,14}$ to the same physical aspects as those cited previously in connection with the suppression of the plasma collapse process near an $X$-type magnetic neutral point in Hall MHD, when Hall effects are sufficiently strong.

\section{DISCUSSION}

In this paper, we have considered Hall MHD properties near a 2D $X$-type magnetic neutral line in the steady state via heuristic as well as rigorous developments. The Hall effects are shown to be able to sustain the hyperbolicity of the magnetic field (and hence a more open $X$-point configuration) near the neutral line in the steady Hall MHD state. This development also shows that the electron-hyperresistivity effects are similar to that of ion-viscosity $v$ and allow for the possibility of an $X$-point magnetic field configuration (see Appendix B), as found also by Chacón et al. ${ }^{13}$ The heuristic development, however, turns out to be useful in providing insight into the lack of dependence of the reconnection rate on the mechanism breaking the frozen-in condition of the magnetic field lines in the electron fluid. The latter result can be understood in terms of the ability of the ions and electrons to transport equal amounts of magnetic flux per unit time out of the reconnection region. The time-dependent Hall MHD problem shows that the Hall effect, when sufficiently strong, can indeed quench the finite-time singularity exhibited in ideal MHD. An $X$-type magnetic field configuration allows the outflow region to open up and eliminates the bottleneck situation limiting the outflow in an elongated current-sheet geometry and hence provides for a mechanism to enhance the reconnection rate in the Hall regime (Shay et al. ${ }^{5}$ ).

\section{ACKNOWLEDGMENTS}

Most of this work was carried out during the course of a visiting appointment at Los Alamos National Laboratory. I acknowledge with gratitude the stimulating interactions 
and discussions with Dr. Luis Chacón that led to this work. My thanks are due to Dr. Michael Shay for helpful communications and Dr. Michael Johnson for helpful discussions. I am very thankful to the referee for his detailed and helpful criticism that led to a clearer development.

\section{APPENDIX A: STABILITY OF THE EQUILIBRIUM SOLUTION OF TIME-DEPENDENT HALL MHD}

Upon including a higher-degree term (which may be viewed as a next term in the Taylor expansion near the magnetic neutral point) for the magnetic stream function $\psi$, we have

$$
\psi(x, y, t)=k \alpha(t) x^{2}-\beta(t) y^{2}+\mu(t) x^{2} y^{2} .
$$

Equation (58) then yields in the nonresistive case,

$$
\dot{\mu}(t)=0 \text { or } \mu(t)=\text { const }=\mu_{0} .
$$

The out-of-plane component of the current density $\mathbf{J}$ is then given by

$$
J_{z}=-\nabla^{2} \psi=-2(k \alpha-\beta)-2 \mu_{0}\left(x^{2}+y^{2}\right) .
$$

The localization of $J_{z}$ near the magnetic neutral point therefore requires $\mu_{0}<0$.

Using (A1) and (A2), Eq. (59) yields, in place of (68),

$$
\ddot{C}+8 \sigma \mu_{0}(k \alpha+\beta)=0 .
$$

In order to investigate the stability of the equilibrium solution (76), consider a small perturbation $u$ about this solution, according to

$$
\dot{\gamma}=-\sigma \dot{C}+\dot{u}
$$

and follow its evolution, as per Eqs. (65) and (A4). This gives

$$
\begin{gathered}
\ddot{u}-\sigma \ddot{C}=8\left(k^{2}+1\right) u+2\left(k^{2}-1\right), \\
\ddot{C}+16 \sigma \mu_{0}(k-1) u=-8 \sigma \mu_{0}(k+1),
\end{gathered}
$$

from which

$$
\begin{gathered}
\ddot{u}+16\left[\sigma^{2} \mu_{0}(k-1)-\left(\frac{k^{2}+1}{2}\right)\right] u \\
=-8 \sigma^{2} \mu_{0}(k+1)+2\left(k^{2}-1\right) .
\end{gathered}
$$

Equation (A8) shows that, on noting $\mu_{0}<0$ and by prescribing the condition $k<1, u$ remains bounded when the Hall effects are sufficiently strong.

Equation (A4), in conjunction with Eqs. (65)-(67), leads to the following exact invariant [in place of (75)] (the author is indebted to Kyle Reger for this observation)

$$
(\dot{\gamma}+\sigma \dot{C})^{2}-\left(k^{2} \alpha^{2}+\beta^{2}\right)+8 \sigma^{2} \mu_{0}(k \alpha-\beta)=\text { const. }
$$

Equation (A9) indicates a somewhat weakening of the runaway behavior of the time-dependent solutions upon including a higher-degree term in $\psi$ in the Hall MHD case.

\section{APPENDIX B: ELECTRON-HYPERRESISTIVITY EFFECTS IN STEADY HALL MHD}

On including the effects of ion-viscosity and electronhyperresistivity, Eqs. (12)-(15) become

$$
\begin{gathered}
\frac{\partial \psi}{\partial t}+[\psi, \phi]+\sigma[b, \psi]=\hat{\eta} \nabla^{2} \psi+\eta_{h} \nabla^{4} \psi \\
\frac{\partial b}{\partial t}+[b, \phi]+\sigma\left[\psi, \nabla^{2} \psi\right]+[\psi, w]=\hat{\eta} \nabla^{2} b+\eta_{h} \nabla^{4} b \\
\frac{\partial}{\partial t}\left(\nabla^{2} \phi\right)+\left[\nabla^{2} \phi, \phi\right]+\left[\psi, \nabla^{2} \psi\right]=v \nabla^{4} \phi \\
\frac{\partial w}{\partial t}+[w, \phi]+[\psi, b]=v \nabla^{2} w
\end{gathered}
$$

where $\eta_{h}$ is the electron hyper-resistivity coefficient.

Following through the development outlined in Sec. IV, we have in place of Eqs. (42), (43), and (46), respectively,

$$
\begin{aligned}
& \psi_{30} \phi_{01}+2 \psi_{20} \phi_{11}+\psi_{10} \phi_{21}-\psi_{21} \phi_{10}-2 \psi_{11} \phi_{20}-\psi_{01} \phi_{30} \\
& +\sigma\left(b_{30} \psi_{01}+2 b_{20} \psi_{11}+b_{10} \psi_{21}-b_{21} \psi_{10}-2 b_{11} \psi_{20}-b_{01} \psi_{30}\right) \\
& =\hat{\eta}\left(\psi_{40}+\psi_{22}\right)+\eta_{h}\left(\psi_{60}+\psi_{24}\right), \\
& \psi_{12} \phi_{01}+2 \psi_{11} \phi_{02}+\psi_{10} \phi_{03}-\psi_{03} \phi_{10}-2 \psi_{02} \phi_{11}-\psi_{01} \phi_{12} \\
& + \\
& +\sigma\left(b_{12} \psi_{01}+2 b_{11} \psi_{02}+b_{10} \psi_{03}-b_{03} \psi_{10}-2 b_{02} \psi_{11}-b_{01} \psi_{12}\right) \\
& =\hat{\eta}\left(\psi_{22}+\psi_{04}\right)+\eta_{h}\left(\psi_{42}+\psi_{06}\right), \\
& b_{21} \phi_{01}+b_{20} \phi_{02}+b_{10} \phi_{12}-b_{12} \phi_{10}-b_{02} \phi_{20}-b_{01} \phi_{21} \\
& \quad+\sigma\left[\psi_{21}\left(\psi_{21}+\psi_{03}\right)+\psi_{20}\left(\psi_{22}+\psi_{04}\right)+\psi_{10}\left(\psi_{32}+\psi_{14}\right)\right. \\
& \left.\quad-\psi_{12}\left(\psi_{30}+\psi_{12}\right)-\psi_{02}\left(\psi_{40}+\psi_{22}\right)-\psi_{01}\left(\psi_{41}+\psi_{23}\right)\right] \\
& \quad+\psi_{21} w_{01}+\psi_{20} w_{02}+\psi_{10} w_{12}-\psi_{12} w_{10}-\psi_{02} w_{20}-\psi_{01} w_{21} \\
& =\hat{\eta}\left(b_{31}+b_{13}\right)+\eta_{h}\left(b_{51}+b_{15}\right) .
\end{aligned}
$$

Using (39) and evaluating Eqs. (B5)-(B7) at the origin,

$$
\begin{gathered}
\hat{\eta}\left(\Psi_{40}+\Psi_{22}\right)+\eta_{h}\left(\Psi_{60}+\Psi_{24}\right)=2 \Psi_{20}\left(\Phi_{11}-\sigma B_{11}\right), \\
\hat{\eta}\left(\Psi_{22}+\Psi_{04}\right)+\eta_{h}\left(\Psi_{42}+\Psi_{06}\right)=-2 \Psi_{02}\left(\Phi_{11}-\sigma B_{11}\right),
\end{gathered}
$$

$$
\begin{aligned}
& \hat{\eta}\left(B_{31}+B_{13}\right)+\eta_{h}\left(B_{51}+B_{15}\right) \\
& \quad=\sigma\left[\Psi_{20}\left(\Psi_{22}+\Psi_{04}\right)-\Psi_{02}\left(\Psi_{40}+\Psi_{22}\right)\right] \\
& \quad+\Psi_{20} W_{02}-\Psi_{02} W_{20} .
\end{aligned}
$$

Using Eqs. (B9) and (B10), Eq. (51) gives

$$
\begin{aligned}
4\left(\Phi_{11}-\sigma B_{11}\right) \Psi_{20} \Psi_{02}= & -v \hat{\eta}\left(\Phi_{51}+2 \Phi_{33}+\Phi_{15}\right) \\
& -\eta_{h}\left(\Psi_{60}+\Psi_{42}+\Psi_{24}+\Psi_{06}\right) .
\end{aligned}
$$

It is of interest to note that the right hand side in Eq. (B11) comes from the term

$$
\left(\frac{\partial^{2}}{\partial x^{2}}+\frac{\partial^{2}}{\partial y^{2}}\right)\left[v \hat{\eta}\left(\frac{\partial^{4}}{\partial x^{3} \partial y}+\frac{\partial^{4}}{\partial x \partial y^{3}}\right) \phi+\eta_{h}\left(\frac{\partial^{4}}{\partial x^{4}}+\frac{\partial^{4}}{\partial y^{4}}\right) \psi\right]
$$


which highlights the similarity between the ion-viscosity and electron-hyperresistivity contributions-both allow the possibility of a $X$-point magnetic field configuration.

${ }^{1}$ A. Clark, Phys. Fluids 7, 1299 (1964).

${ }^{2}$ B. U. O. Sonnerup, in Solar System Plasma Physics, edited by L. J. Lanzerotti, C. F. Kennel, and E. N. Parker (North-Holland, Amsterdam, 1979), p. 45.

${ }^{3}$ J. C. Dorelli and J. Birn, J. Geophys. Res. 108, 1133, doi:10.1029/ 2002JA009180 (2003).

${ }^{4}$ D. A. Knoll and L. Chacón, Phys. Rev. Lett. 96, 135001 (2006).

${ }^{5}$ M. A. Shay, J. F. Drake, B. N. Rogers, and R. E. Denton, Geophys. Res. Lett. 26, 2163, doi:10.1029/1999GL900481 (1999).

${ }^{6}$ B. N. Rogers, R. E. Denton, J. F. Drake, and M. A. Shay, Phys. Rev. Lett. 87, 195004 (2001).

${ }^{7}$ R. Fitzpatrick, Phys. Plasmas 11, 937 (2004).

${ }^{8}$ P. A. Sweet, in Electromagnetic Phenomena in Cosmic Physics, edited by B. Lehnert (Cambridge University Press, Cambridge, 1958), p. 123.

${ }^{9}$ E. N. Parker, J. Geophys. Res. 62, 509, doi:10.1029/JZ062i004p00509 (1957).

${ }^{10}$ H. E. Petschek, in AAS/NASA Symposium on the Physics of Solar Flares (NASA, Washington, DC, 1964), p. 425.

${ }^{11}$ W. Daughton, J. Scudder, and H. Karimabadi, Phys. Plasmas 13, 072101 (2006).

${ }^{12}$ H. Karimabadi, W. Daughton, and J. Scudder, Geophys. Res. Lett. 34, L13104, doi:10.1029/2007GL030306 (2007).

${ }^{13}$ L. Chacón, A. N. Simakov, and A. Zocco, Phys. Rev. Lett. 99, 235001 (2007).

${ }^{14}$ M. A. Shay, J. F. Drake, and M. Swisdak, Phys. Rev. Lett. 99, 155002 (2007).

${ }^{15}$ A. Frank, S. G. Bugrov, and V. S. Markov, Phys. Plasmas 15, 092102 (2008).

${ }^{16}$ S. I. Syrovatskii, J. Sov. Astron. 10, 270 (1966).

${ }^{17}$ M. S. Uberoi, Phys. Fluids 6, 1379 (1963).

${ }^{18}$ S. Chapman and P. C. Kendall, Proc. Roy. Soc. London A 271, 435 (1963).

${ }^{19}$ B. K. Shivamoggi, Phys. Fluids 29, 769 (1986).

${ }^{20}$ B. K. Shivamoggi, Phys. Lett. A 258, 131 (1999).

${ }^{21}$ D. K. Rollins and B. K. Shivamoggi, Europhys. Lett. 57, 492 (2002).

${ }^{22}$ D. K. Rollins and B. K. Shivamoggi, Phys. Lett. A 366, 97 (2007).

${ }^{23}$ A. G. Frank, Proc. (Tr.) P.N.Lebedev Phys. Inst. 74, 108 (1974).

${ }^{24}$ F. Brunnel, T. Tajima, and J. M. Dawson, Phys. Rev. Lett. 49, 323 (1982).

${ }^{25}$ B. K. Shivamoggi, Europhys. Lett. 85, 25001 (2009).

${ }^{26}$ Y. E. Litvinenko, Phys. Plasmas 14, 112303 (2007).
${ }^{27}$ B. K. Shivamoggi, Phys. Lett. A 373, 708, (2009).

${ }^{28}$ B. K. Shivamoggi, arXiv:0801.3403v4 (2008).

${ }^{29}$ M. E. Mandt, R. E. Denton, and J. F. Drake, Geophys. Res. Lett. 21, 73, doi:10.1029/93GL03382 (1994).

${ }^{30}$ M. A. Shay and J. F. Drake, Geophys. Res. Lett. 25, 3759, doi:10.1029/ 1998GL900036 (1998).

${ }^{31}$ A. N. Simakov and L. Chacón, Phys. Rev. Lett. 101, 105003 (2008).

${ }^{32}$ L. M. Malyshkin, Phys. Rev. Lett. 101, 225001 (2008).

${ }^{33}$ B. Coppi, G. Laval, and R. Pellat, Phys. Rev. Lett. 16, 1207 (1966).

${ }^{34}$ A. Zocco, L. Chacón and A. N. Simakov, Phys. Plasmas 16, 110703 (2009).

${ }^{35}$ L. M. Malyshkin, Phys. Rev. Lett. 103, 235004 (2009).

${ }^{36}$ B. P. Sullivan, A. Bhattacharjee, and Y. M. Huang, Phys. Plasmas 16, 102111 (2009).

${ }^{37}$ J. F. Drake, M. A. Shay and M. Swisdak, Phys. Plasmas 15, 042306 (2008).

${ }^{38}$ T. Tsuda and M. Ugai, J. Plasma Phys. 17, 337 (1977).

${ }^{39}$ S. W. H. Cowley, J. Plasma Phys. 14, 475 (1975).

${ }^{40}$ B. K. Shivamoggi, J. Plasma Phys. 31, 333 (1985).

${ }^{41}$ T. Terasawa, Geophys. Res. Lett. 10, 475 (1983).

${ }^{42}$ Y. Ren, M. Yamada, S. Gerhardt, H. Ji, R. Kulsrud, and A. Kuritsyn, Phys. Rev. Lett. 95, 055003 (2005).

${ }^{43}$ M. Fujimoto, M. S. Nakamura, I. Shinohara, T. Nagai, T. Mukai, Y. Saito, T. Yamamoto, and S. Kokubun, Geophys. Res. Lett. 24, 2893, doi:10.1029/97GL02821 (1997).

${ }^{44}$ T. Nagai, I. Shinohara, M. Fujimoto, M. Hoshino, Y. Saito, S. Machida, and T. Mukai, J. Geophys. Res. 106, 25929, doi:10.1029/2001JA900038 (2001).

${ }^{45}$ M. Oieroset, T. D. Phan, M. Fujimoto, R. P. Lin, and R. P. Lepping, Nature 412, 414 (2001).

${ }^{46}$ B. K. Shivamoggi, Phys. Plasmas 16, 052111 (2009).

${ }^{47}$ B. K. Shivamoggi, in Quantum Vortex Dynamics and Superfluid Turbulence, edited by C. Barenghi, R. Donnelly, and W. F. Vinen (Springer-Verlag, Berlin, 2001).

${ }^{48}$ B. K. Shivamoggi, Euro. Phys. J. B 49, 483 (2006).

${ }^{49}$ B. K. Shivamoggi, Europhys. Lett. 83, 55002 (2008).

${ }^{50}$ M. A. Shay, J. F. Drake, M. Swisdak, and B. N. Rogers, Phys. Plasmas 11, 2199 (2004).

${ }^{51}$ J. M. Urrutia, M. C. Griskey, and R. M. Stenzel, Bull. Am. Phys. Soc. 46(8), 87 (2001).

${ }^{52}$ B. U. O. Sonnerup, G. Paschmann, I. Papamastorakis, N. Sckopke, G. Haerendel, S. J. Barne, S. R. Asbridge, J. I. Goshing, and C. T. Russell, J. Geophys. Res. 86, 10049, doi:10.1029/JA086iA12p10049 (1981).

${ }^{53}$ D. H. Fairfield, E. W. Hones, Jr., and C. I. Meng, J. Geophys. Res. 86, 11189, doi:10.1029/JA086iA13p11189 (1981). 